Original Research Paper

\title{
Automatic Detection of the End-Diastolic and End-Systolic from 4D Echocardiographic Images
}

\author{
${ }^{1}$ Anas A. Abboud, ${ }^{1}$ Rahmita Wirza Rahmat, ${ }^{2}$ Suhaini Bin Kadiman, ${ }^{3}$ Mohd Zamrin Bin Dimon, \\ ${ }^{1}$ Lili Nurliyana, ${ }^{4}$ M. Iqbal Saripan and ${ }^{1}$ Hasan H. Khaleel \\ ${ }^{I}$ Department of Computer Science and Information Technology, UPM, 43400, Serdang, Malaysia \\ ${ }^{2}$ National Heart Institute (IJN), No 145 Jalan Tun Razak, 50400 Kuala Lumpur, Malaysia \\ ${ }^{3}$ Faculty of Medicine, Universiti Teknologi MARA(UiTM), 47000, Sungai Buloh,Malaysia \\ ${ }^{4}$ Department of Computer and Communication Systems Engineering, Faculty of Engineering, UPM, 43400, Serdang, Malaysia
}

\author{
Article history \\ Received: 05-03-2014 \\ Revised: 26-05-2014 \\ Accepted: 09-08-2014 \\ Corresponding Author: \\ Anas A. Abboud \\ Department of Computer Science \\ and Information Technology, \\ University Putra Malaysia, 43400, \\ Serdang, Malaysia \\ Email: anasalabousyupm@gmail.com
}

\begin{abstract}
Accurate detection of the End-Diastolic (ED) and EndSystolic (ES) frames of a cardiac cycle are significant factors that may affect the accuracy of abnormality assessment of a ventricle. This process is a routine step of the ventricle assessment procedure as most of the time in clinical reports many parameters are measured in these two frames to help in diagnosing and dissection making. According to the previous works the process of detecting the ED and ES remains a challenge in that the ED and ES frames for the cavity are usually determined manually by review of individual image phases of the cavity and/or tracking the tricuspid valve. The proposed algorithm aims to automatically determine the ED and ES frames from the four Dimensional Echocardiographic images (4DE) of the Right Ventricle (RV) from one cardiac cycle. By computing the area of three slices along one cardiac cycle and selecting the maximum area as the ED frame and the minimum area as the ES frame. This method gives an accurate determination for the ED and ES frames, hence avoid the need for time consuming, expert contributions during the process of computing the cavity stroke volume.
\end{abstract}

Keywords: End-Diastolic (ED) and End-Systolic (ES), Echocardiography Image, Stroke Volume, Right Ventricle (RV), Left Ventricle (LV), Three Dimensional Echocardiography (3DE), Proposed Method, QLAB System, Automatic Algorithm, Wall Motion

\section{Introduction}

Accurate detection of the End-Distally (ED) and EndSystolic (ES) stages of a cardiac cycle is a significant factor that can affect the accuracy of abnormality assessment of a ventricle (Ostenfeld et al., 2012; Darvishi et al., 2012). This process is a routine step of the ventricle assessment procedures, where often, in clinical reports, many parameters can be measured in these two stages to help in diagnosing and decision making (Umberto et al., 2008). However, the process of detecting the ED and ES still a challenge for diagnosis because it was mostly done manually by tracking the cine loop during the clinical procedure, as in
(Niemann et al., 2007) or by optical tracking of the change of ventricle dimensions through watching the individual image faces of the ventricle during the cardiac cycle, as in (Aune et al., 2009; Crean et al., 2011; Dawood et al., 2011).

Tracking the mitral valve motion is another method for detecting the ED and ES frame (Hussein et al., 2011), where the ES stage is defined as the last frame before the opening of the mitral valve while the ED stage is the last frame before the closing for the mitral valve, as reported by (Nosir et al., 1996). In the meanwhile, tracking the mitral valve together with the ventricle volume was presented by (Umberto et al., 2008). ECG signal was used by (Zhu et al., 2009) to 
determine the ED and ES frame of the LV in order to generate a dynamic model of the LV. (Gopal et al., 2007; Chua et al., 2009) also used ECG to determine the ED frame through the R wave of the ECG and the ES frame was determined as the smallest cavity area. The biggest and smallest area of the RV cavity were visually tracked in three views; short axis, four chamber and coronal by (Ostenfeld et al., 2012) to determine the ED and ES cardiac stages.

Gifani et al. (2010) presented an automatic detection method for ED and ES of LV from two and four chamber views using unsupervised learning algorithm (Locally Linear Embedding (LLE)) for three cardiac consecutive cycles. (Shalbaf et al., 2011) performed image registration for echocardiographic images of 6 volunteers, distance computation and finally the classical Multi-Dimensional Scaling (MDS) used to construct low dimensional representation of $2 \mathrm{D} \mathrm{LV}$ images to generate Iso-map. Then, they computed manifold model of seven phases of cardiac cycles to determine ED and ES stages automatically.

(Yuanfang et al., 2011) determined ED and ES stages by tracking the mitral valve motion, based on (Nosir et al., 1996)'s definition for the ED and ES stages. This method used 3D echo image of LV, by binaries and enhancing images in the pre-processing stage of their algorithm. Then, a dilation operation for different resolution scale was performed to avoid the blood flow effect. Finally, the number of connecting regions for 8-neighbore connectivity was computed. This method gives accurate results only with good quality echo image of mitral valve.

All of methods reviewed in this literature are time consuming; therefore, an accurate automatic detection for the ED and ES stages is highly required to assist specialists and interns in their clinical work.

\section{Materials and Methods}

The main goal of the proposed method is to enable automatic detection of the ED and ES frames from one cardiac cycle. This process is one of the steps of the main algorithm for measuring the RV stroke, which proposed automatic measuring for the ventricle stroke volume. Whereby the ventricle stroke volume is the volume of blood ejected from a ventricle at each beat of the heart. This is equal to the difference between the cavity volume at the End-Diastolic stage (EDV) and the cavity volume of at the End-Systolic stage (ESV) of the cardiac cycle according to (Barash et al., 2011). This requires the determination of the ED and ES frames of the cardiac cycle and measuring the cavity volume at the end-diastolic and end-systolic as stated by (Klabunde, 2011).
The cardiac cycle duration (or the number of frames in a cardiac cycle) is different from one patient to another. The ES frame position in the echocardiography video of a cardiac cycle (the ES stage of a cardiac cycle) is also different from one patient to another, as illustrated in Table 1. The ED frame as determined by the experts can be used as the first image in the cardiac cycle. It can also be considered as the frame following closure of the mitral valve, or the frame of the largest cavity in the cardiac cycle.

An automatic detection of the ED-ES frames is proposed, by measuring the area of the segmented cavity of the RV along a cardiac cycle for three slices in the middle region of the RV, as in Equation 1:

$\operatorname{Area}_{s(r)}=\sum_{i=1}^{n} \sum_{j=1}^{m} f(i, j)$, for all $f(i, j)>0$

where, $\operatorname{Area}_{s(r)}$ is the area of the segmented region in slice number $\mathrm{r}$, where $\mathrm{r}=8,9,10$ and $f(i, j)$ is the grayscale of the pixels in the segmented region.

These three slices $(8,9$ and 10) which are located in the middle region of the RV cavity as presented in Fig. 1 , is selected based on analyzing the structure and motion behavior of the 16 slices over a cardiac cycle for the patients in the dataset, of the RV cavity.

The details of selecting these slices are explained in the following sub-section.

\section{Slice Selection}

Selecting the slices from the middle region of the RV cavity is based on two anatomical facts.

First; the free wall area of the RV cavity in the middle region (fractional region) is broader than the inflow and out flow tract as illustrated in Fig. 2 and 3.

Therefore, for fully automatic detection of the ED-ES frame, the middle region of the cavity is sliced for this purpose. The middle region has a close intracardiac boundary along the cardiac cycle, while the inflow and outflow tract is affected by the motion of the valves (opening and closing) during the cardiac cycle. When the valve is closed, the region will be closed and segmentation includes only the intra cavity region.

However, when the valve is open, the segmentation will be growing out of the inflow and/or outflow tract. Figure 3 illustrates the intracardiac boundary of the RV in inflow, middle and out flow regions along different frames of one cardiac cycle video, for a patient.

Second; the amount of changes in the cavity area, in the middle region during the cardiac cycle is higher than the changes of the inflow and outflow tract. To 
experiment with this fact, the area of the 16 slices is computed for one cardiac cycle for one sample patient of the dataset.

Then the standard deviation and variance of the changing for total area of the cavity in each slice are computed for the comparison of each slice in one cardiac cycle. This is illustrated in Table 1-4 respectively and the corresponding diagram in Fig. 46 , respectively for a sample of three patients of the data set.

The Tables and Diagrams clearly show that the middle region is highly affected along the cardiac cycle for which to be a candidate it has to be a good indicator of the ED and ES cardiac cycle frames.

By averaging the three previous samples, it can be concluded that the middle region has the highest fractional region (the region with maximum change in the cavity during the cardiac cycle), this is illustrated in Fig. 7.

\section{Selecting the ED-ES Frames}

In the proposed method, three slices are used to detect the ED and ES frames, as using three frames may provide accurate detection of the ED and ES frames. The diagram in Fig. 8 illustrates the method of detecting the ED and ES frames of the cardiac cycle.

As stated earlier in the proposed method, three slices are used to determine the frames of the ED and the ES stages. The reason for selecting three slices can be justified by computation analysis as in the following steps.
Compute the change in the area of the segmented region in the frame $\rho$ and compare it with the area of the frame $\rho-1$ of the same slice, for each of the three slices, as shown in Table 4.

According to the definition of the ED Frame, the maximum area of the segmented region is tracked from Table 4 . The segmented areas for each of three slices $(8,9$ and 10) in the first row are compared with its area in the second row. We can see that; for the slice number 8 the area is decrease in the second row and for slice number 9 and 10 the area increase in the second row, however the average of the area of the tree slices still the highest in the first row, thus we use the frame number in the first row as the ED stage of the cardiac cycle. Therefore the frame of first row can be considered as the ED frame.

By tracing the changes of the area, the minimum area in each slice falls in different frame numbers. For example in the segmented region area of slice 10, the area decreases in the frame 10,11 , until it reaches the minimum area in frame 12 and it starts to increase again in frame 13, as illustrated in Fig. 9. However, the segmented region area of slice 9 increases in frame 10 , decreases to the minimum in frame 11 and then continues increasing in frames $12,13 \ldots$ and so on. This procedure shown in Table 4 by the arrows.

To satisfy the accuracy of determining the ED and ES frames, the mean of the area is computed for three slices using Equation 2 and Equation 3 and the result is shown in Fig. 10.

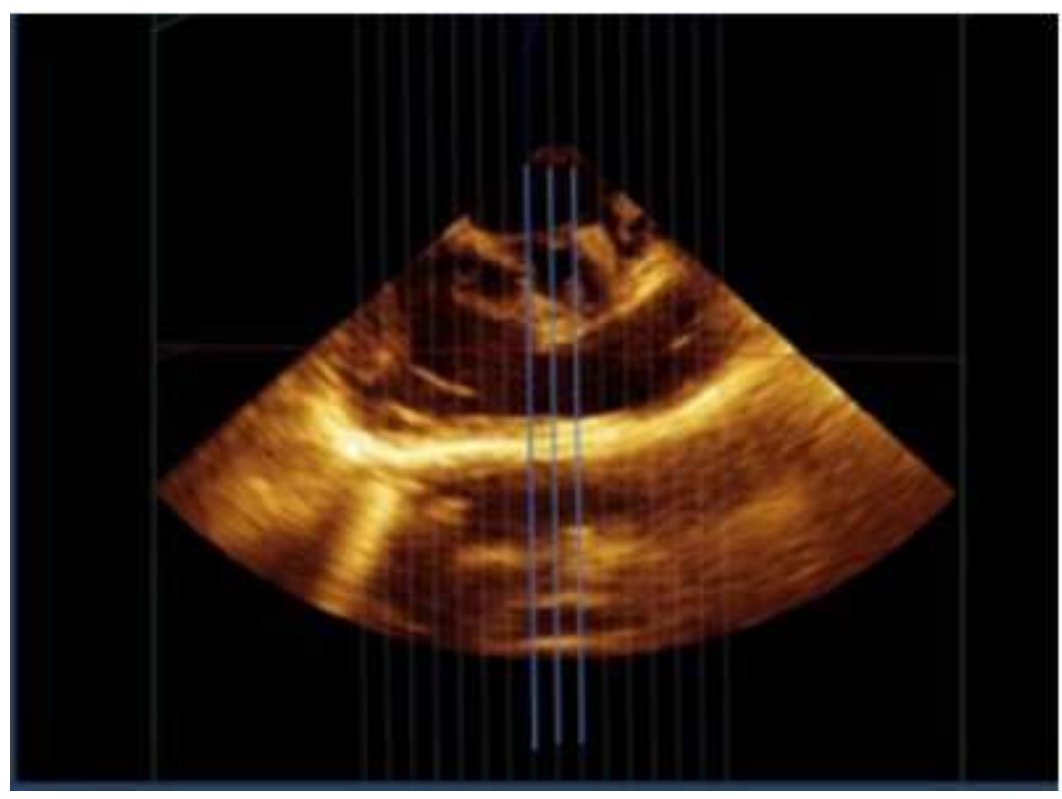

Fig. 1. Three slices from the middle region of the RV cavity. Slice 8, Slice 9 and Slice 10 from left to right 


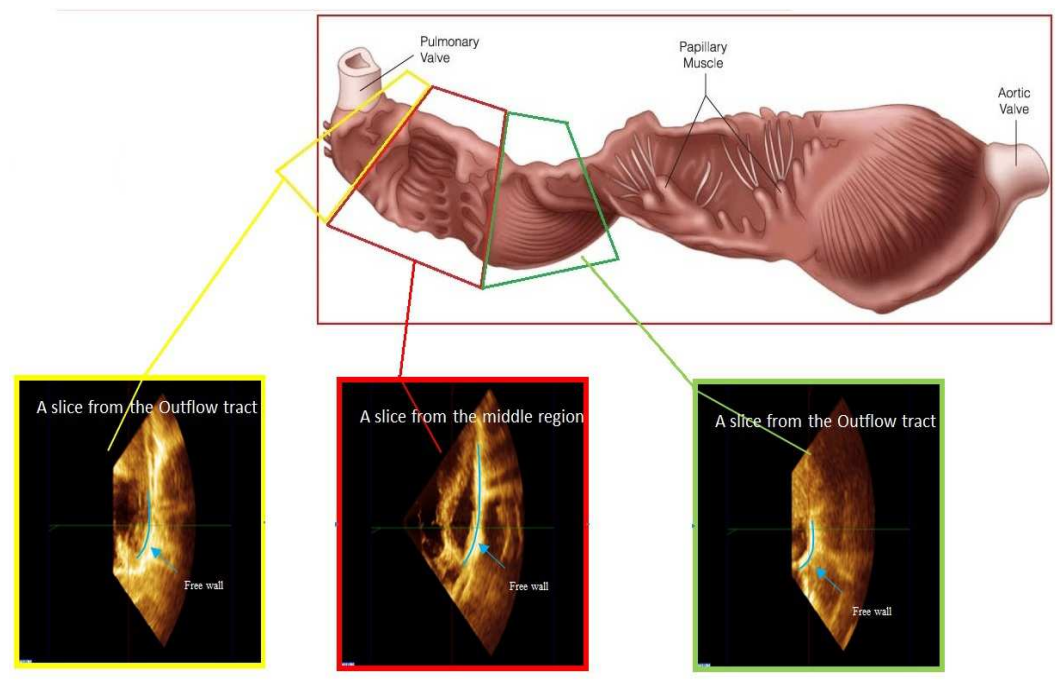

Fig. 2. Right ventricle slices at inflow, middle and outflow regions of RV cavity

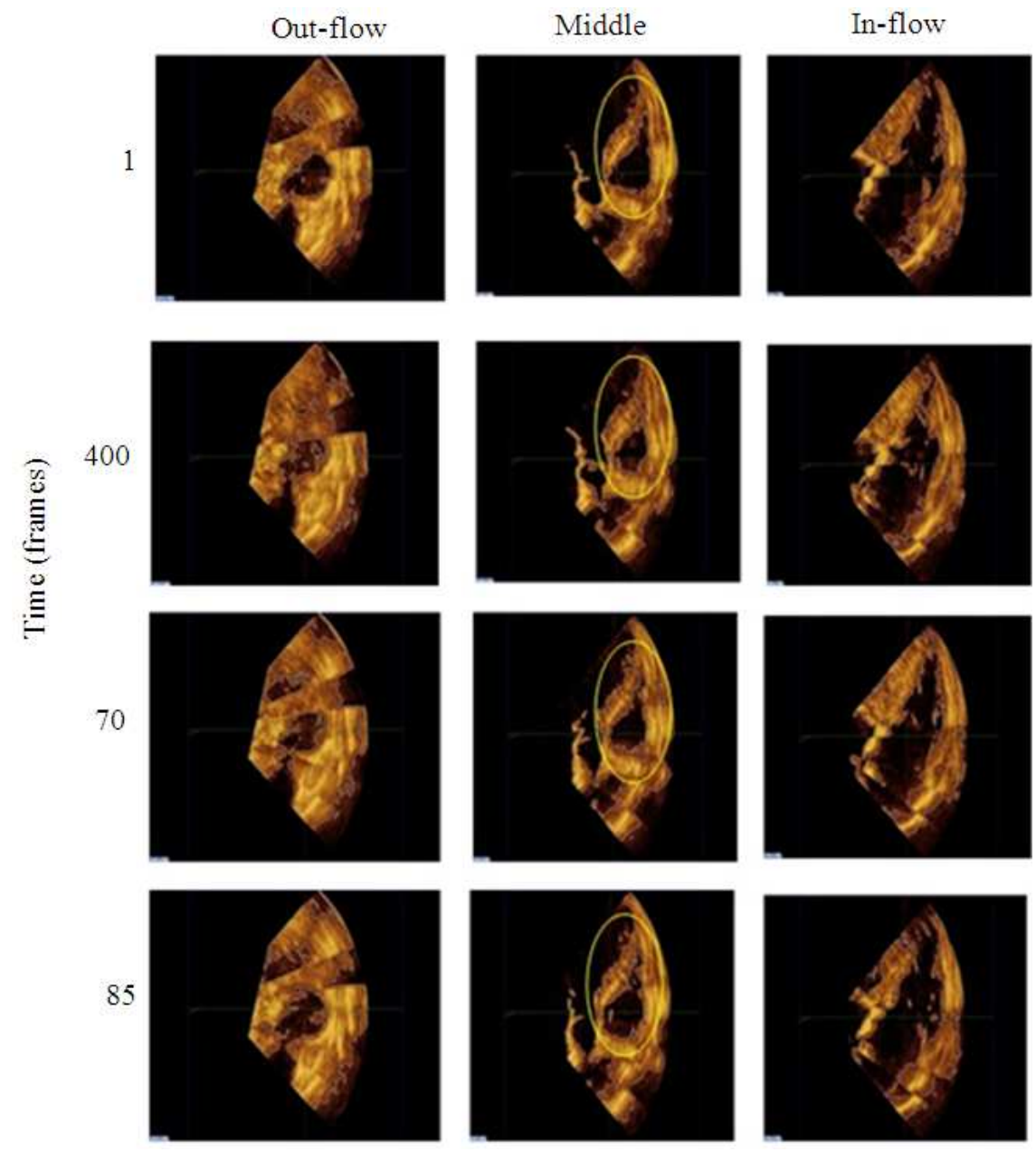

Fig. 3. The three regions (Inflow-Middle-Outflow) of the RV cavity for one patient of the data set, explain the changes in the cavity during a cardiac cycle and the effect of opening the TV and PV. The RV cavity boundary in the middle region (highlighted) is closed in the middle during the cardiac cycle 


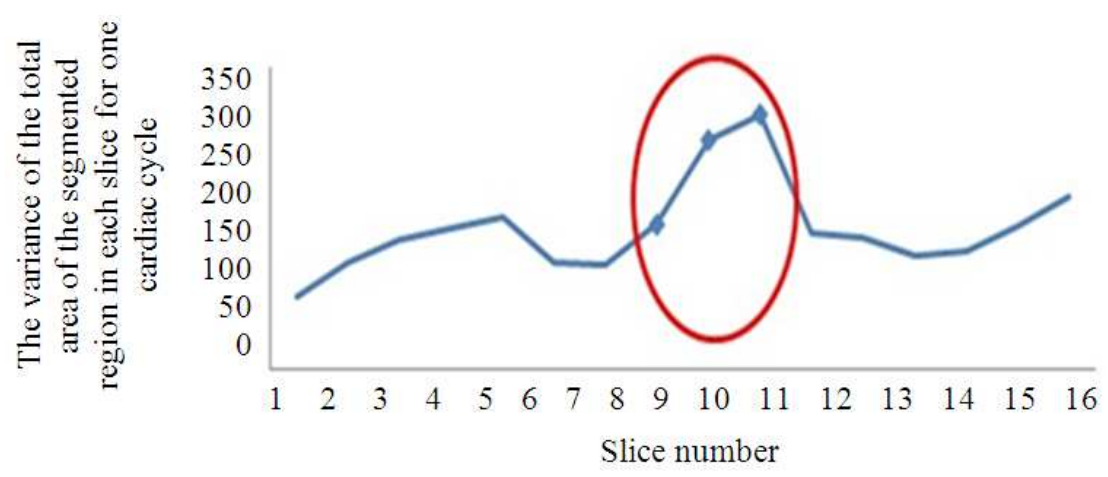

Fig. 4. Diagram of changing in the cavity area in each slice during one cardiac cycle for Patient 1

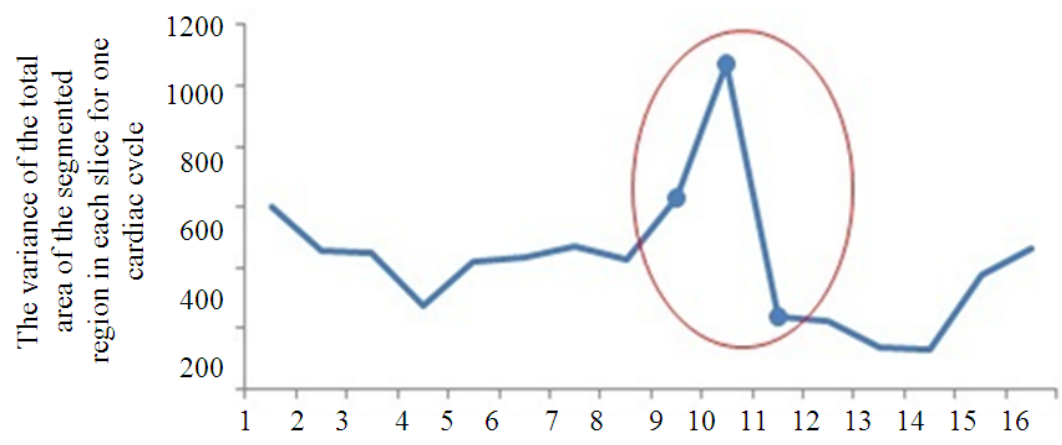

Fig. 5. Diagram of changing in the cavity area in each slice during one cardiac cycle for Patient 2

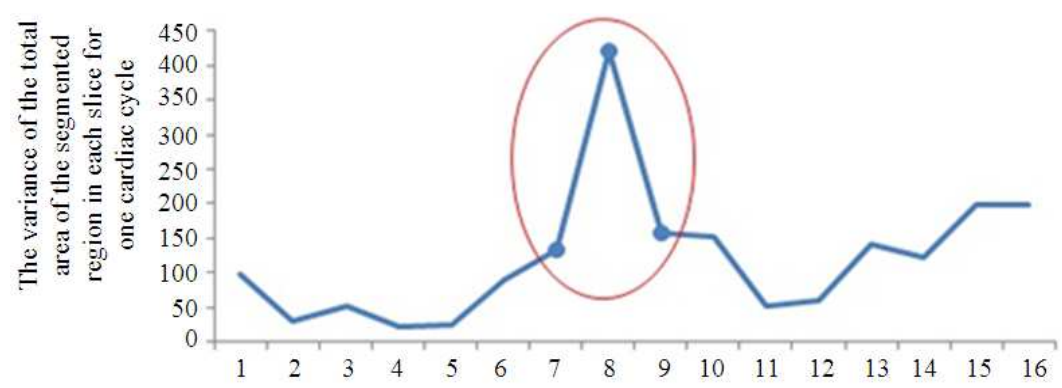

Fig. 6. Diagram of changing in the cavity area in each slice during one cardiac cycle for Patient 3

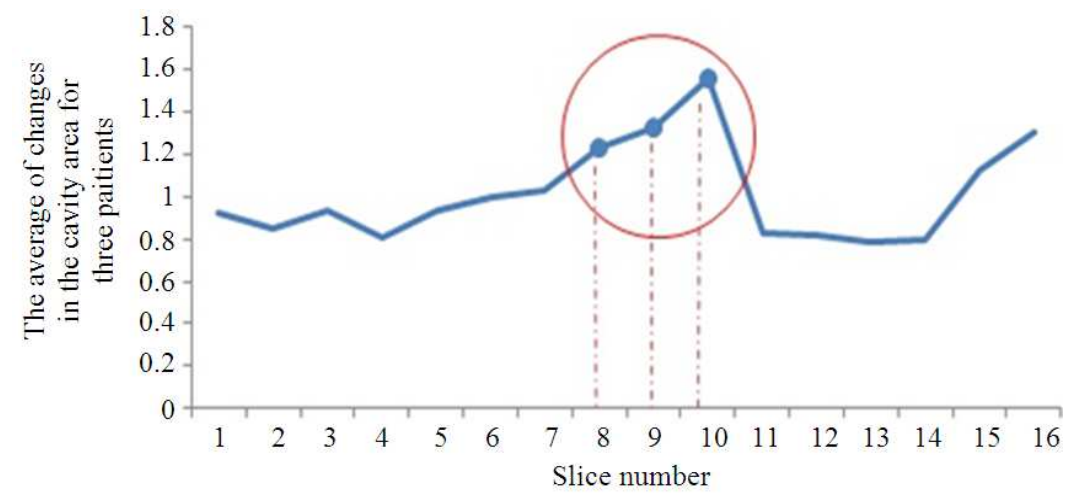

Fig. 7. Average of the standard deviation of the three patients, illustrating that the middle region is the greatest changing region affected in the cavity during the cardiac cycle 


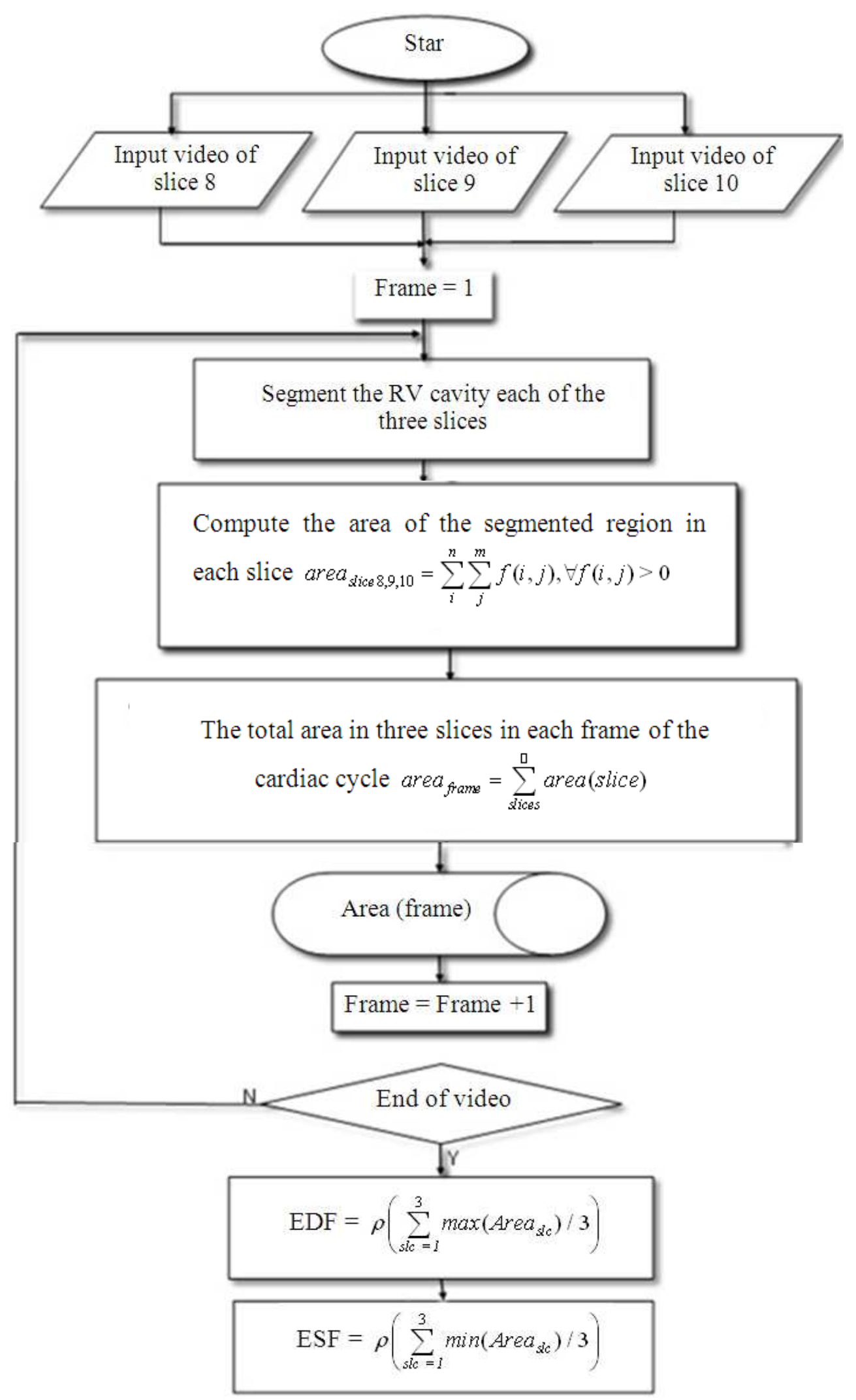

Fig. 8. Diagram of the method of the determination of ED and ES 


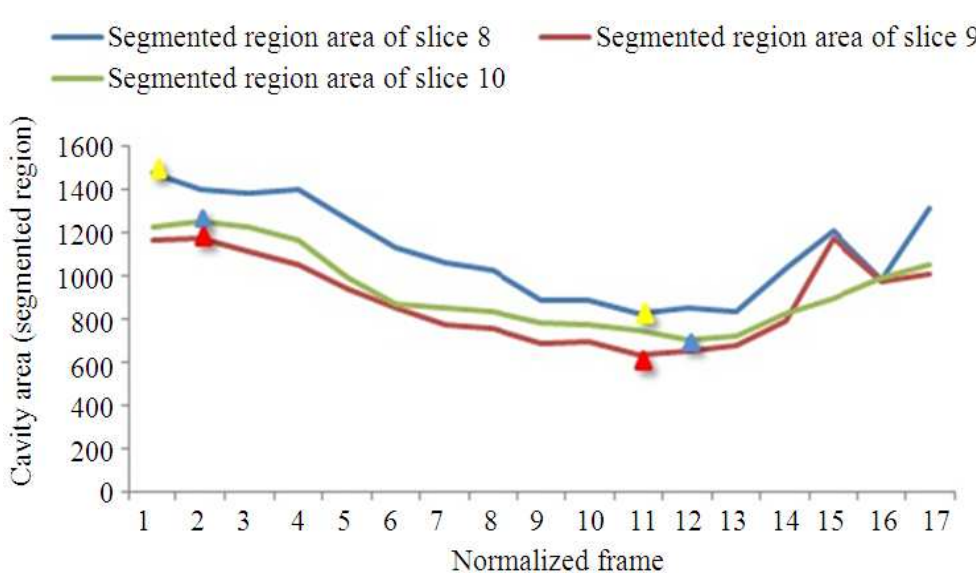

Fig. 9. The area of three slices $(8,9$ and 10$)$ during the cardiac cycle

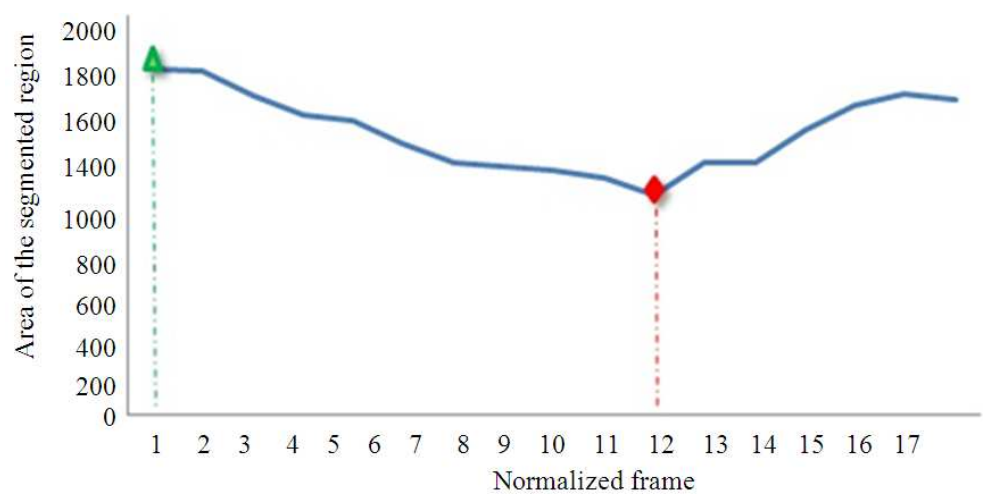

Fig. 10. The mean of areas for the three slices $(8,9$ and 10) and the frames, the maximum area indicates the ED frame and the minimum area indicates the ES frame

Table 1 . The changes of the cavity area for Patient 1 , during one cardiac cycle in each slice ( $\mathrm{Si}$ ) are the slices I $=1$ to $16, \mathrm{Ni}$ is the frames of one cardiac cycle video

\begin{tabular}{|c|c|c|c|c|c|c|c|c|c|c|c|c|c|c|c|c|}
\hline N.Frame & S1 & $\mathrm{S} 2$ & S3 & S4 & S5 & S6 & S7 & S8 & S9 & $\mathrm{S} 10$ & S11 & $\mathrm{S} 12$ & $\mathrm{~S} 13$ & S14 & S15 & S16 \\
\hline N1 & 820 & 927 & 982 & 950 & 1195 & 909 & 817 & 960 & 1120 & 1247 & 1041 & 1233 & 1287 & 1435 & 928 & 1263 \\
\hline N2 & 700 & 802 & 881 & 998 & 1200 & 996 & 884 & 852 & 1092 & 1364 & 1091 & 1239 & 1311 & 1347 & 1002 & 1277 \\
\hline N3 & 726 & 745 & 832 & 972 & 1093 & 990 & 843 & 754 & 902 & 1064 & 1092 & 1111 & 1182 & 1264 & 916 & 900 \\
\hline N4 & 766 & 766 & 766 & 851 & 1013 & 915 & 804 & 658 & 679 & 973 & 854 & 1078 & 1140 & 1188 & 846 & 830 \\
\hline N5 & 796 & 664 & 660 & 726 & 906 & 850 & 718 & 592 & 584 & 916 & 756 & 953 & 1094 & 1054 & 603 & 735 \\
\hline N6 & 774 & 620 & 579 & 652 & 820 & 830 & 670 & 529 & 479 & 839 & 664 & 1017 & 1090 & 981 & 597 & 696 \\
\hline N7 & 685 & 573 & 530 & 630 & 790 & 801 & 638 & 542 & 416 & 485 & 688 & 973 & 926 & 1022 & 552 & 658 \\
\hline N8 & 654 & 548 & 536 & 657 & 772 & 783 & 586 & 490 & 510 & 504 & 726 & 1176 & 1302 & 1289 & 553 & 706 \\
\hline N9 & 599 & 554 & 543 & 687 & 787 & 807 & 599 & 492 & 402 & 508 & 788 & 1211 & 1348 & 1300 & 501 & 678 \\
\hline N10 & 603 & 551 & 518 & 580 & 721 & 778 & 584 & 508 & 388 & 498 & 765 & 1164 & 1404 & 1347 & 592 & 738 \\
\hline N11 & 603 & 556 & 534 & 520 & 779 & 608 & 577 & 413 & 344 & 446 & 632 & 843 & 1358 & 1290 & 597 & 742 \\
\hline $\mathrm{N} 12$ & 634 & 585 & 614 & 504 & 738 & 566 & 565 & 392 & 354 & 855 & 695 & 805 & 1125 & 1338 & 620 & 800 \\
\hline N13 & 684 & 667 & 620 & 596 & 637 & 706 & 484 & 419 & 579 & 855 & 679 & 822 & 1266 & 1151 & 609 & 742 \\
\hline N14 & 858 & 847 & 785 & 792 & 881 & 763 & 646 & 607 & 824 & 940 & 825 & 996 & 1170 & 1131 & 778 & 907 \\
\hline Variance & 7255 & 15713 & 23065 & 27338 & 31697 & 15764 & 14836 & 28407 & 70943 & 87740 & 24971 & 23251 & 17619 & 19059 & 27425 & 39833 \\
\hline STDV & 85 & 125 & 152 & 165 & 178 & 126 & 122 & 169 & 266 & 296 & 158 & 152 & 133 & 138 & 166 & 200 \\
\hline
\end{tabular}

$E D F=\rho \frac{\sum_{s l c=1}^{3} \operatorname{Max}\left(\operatorname{area}_{s l c}\right)}{3}$

$$
E S F=\rho \frac{\sum_{s l c=1}^{3} \min \left(\operatorname{area}_{s l c}\right)}{3}
$$

where, is an indicator of the position of the frames in the cardiac cycle video, as declared in Table 6, EDF is the end diastolic frame, ESF is the end systolic frame and Area $_{\text {slc }}$ is the area of the segmented region of the cavity in the slice.

For the sample data used in Fig. 9 and 10, the number of frames in one cardiac cycle is 68 frames. According to the computed segmented area and the frames, ES frame $\rho=11$ is frame number 41 in the video. 
Table 2. The changes of slice area for Patient 2, during one cardiac cycle $(\mathrm{Si})$ are the slices $\mathrm{I}=1$ to $16, \mathrm{Ni}$ is the frames of one cardiac cycle video

\begin{tabular}{|c|c|c|c|c|c|c|c|c|c|c|c|c|c|c|c|c|}
\hline & S1 & S2 & S3 & S4 & S5 & S6 & S7 & S8 & S9 & S10 & S11 & $\mathrm{S} 12$ & S13 & S14 & S15 & S16 \\
\hline N1 & 1763 & 1728 & 1678 & 1559 & 1776 & 1733 & 1854 & 1864 & 1854 & 2241 & 2620 & 2899 & 2615 & 2265 & 1950 & 1600 \\
\hline N2 & 1794 & 1739 & 1713 & 1573 & 1805 & 1689 & 1830 & 1458 & 1499 & 2208 & 2568 & 2871 & 2621 & 2273 & 2015 & 1695 \\
\hline N3 & 1860 & 1751 & 1694 & 1562 & 1780 & 1668 & 1720 & 1406 & 1515 & 2141 & 2508 & 2800 & 2461 & 2026 & 1724 & 1478 \\
\hline N4 & 1721 & 1649 & 1554 & 1509 & 1679 & 1582 & 1635 & 1329 & 1385 & 1988 & 2426 & 2716 & 2445 & 2052 & 1750 & 1573 \\
\hline N5 & 1540 & 1495 & 1346 & 1360 & 1454 & 1475 & 1608 & 1611 & 1248 & 1475 & 2261 & 2690 & 2453 & 2044 & 1813 & 1648 \\
\hline N6 & 1440 & 1363 & 1278 & 1318 & 1386 & 1338 & 1445 & 1511 & 1162 & 1293 & 2183 & 2578 & 2393 & 1971 & 1643 & 1562 \\
\hline N7 & 1353 & 1267 & 1231 & 1264 & 1328 & 1271 & 1335 & 1394 & 1089 & 1318 & 2225 & 2459 & 2334 & 1966 & 1589 & 1411 \\
\hline N8 & 1278 & 1230 & 1177 & 1206 & 1280 & 1228 & 1328 & 1373 & 1056 & 1251 & 2182 & 2494 & 2293 & 1884 & 1540 & 1396 \\
\hline N9 & 1216 & 1173 & 1167 & 1175 & 1262 & 1210 & 1279 & 1365 & 1056 & 1429 & 2246 & 2459 & 2262 & 1968 & 1470 & 1374 \\
\hline N10 & 1175 & 1170 & 1145 & 1110 & 1262 & 1223 & 1276 & 1312 & 1023 & 1463 & 2169 & 2469 & 2273 & 1952 & 1501 & 1144 \\
\hline N11 & 1168 & 1214 & 1180 & 1160 & 1305 & 1280 & 1247 & 936 & 1052 & 1777 & 2137 & 2429 & 2271 & 1943 & 1463 & 1068 \\
\hline N12 & 1105 & 1178 & 1191 & 1146 & 1314 & 1323 & 1209 & 1305 & 1269 & 1730 & 2175 & 2479 & 2335 & 1952 & 1468 & 980 \\
\hline N13 & 1222 & 1202 & 1190 & 1199 & 1342 & 1114 & 1253 & 1291 & 1250 & 1631 & 2253 & 2569 & 2256 & 1888 & 1318 & 1061 \\
\hline N14 & 1390 & 1328 & 1328 & 1256 & 1459 & 1235 & 1461 & 1406 & 1452 & 1706 & 2277 & 2579 & 2348 & 1881 & 1436 & 1330 \\
\hline N15 & 1564 & 1439 & 1484 & 1417 & 1600 & 1567 & 1578 & 1575 & 1612 & 1813 & 2342 & 2674 & 2379 & 1990 & 1528 & 1279 \\
\hline N16 & 1604 & 1500 & 1558 & 1482 & 1660 & 1626 & 1621 & 1616 & 1719 & 1946 & 2427 & 2649 & 2442 & 2041 & 1681 & 1364 \\
\hline N17 & 1638 & 1536 & 1610 & 1515 & 1719 & 1685 & 1730 & 1719 & 1325 & 2053 & 2511 & 2764 & 2518 & 2067 & 1819 & 1498 \\
\hline Var. & 59980 & 45367 & 44613 & 27405 & 41775 & 43767 & 47212 & 42437 & 63179 & 107029 & 24103 & 22794 & 13406 & 12864 & 37336 & 46398 \\
\hline STDV & 244 & 213 & 211 & 165 & 204 & 209 & 217 & 206 & 251 & 327 & 155 & 150 & 115 & 113 & 193 & 215 \\
\hline
\end{tabular}

Table 3. The changes of slice area for Patient 3, during one cardiac cycle $(\mathrm{Si})$ are the slices $\mathrm{I}=1$ to $16, \mathrm{Ni}$ is the frames of one cardiac cycle video

\begin{tabular}{|c|c|c|c|c|c|c|c|c|c|c|c|c|c|c|c|c|}
\hline & $\mathrm{S} 1$ & S2 & S3 & S4 & S5 & S6 & S7 & S8 & S9 & $\mathrm{S} 10$ & S11 & $\mathrm{S} 12$ & $\mathrm{~S} 13$ & S14 & S15 & S16 \\
\hline N1 & 2027 & 1488 & 968 & 1127 & 932 & 1105 & 1317 & 1502 & 1812 & 1874 & 1928 & 1894 & 2101 & 2061 & 1947 & 1219.00 \\
\hline N2 & 1944 & 1482 & 971 & 1124 & 941 & 1106 & 1298 & 1530 & 1579 & 1678 & 1942 & 1922 & 2322 & 2052 & 1915 & 1160.00 \\
\hline N3 & 1693 & 1493 & 949 & 1132 & 970 & 1121 & 1337 & 1526 & 1552 & 1781 & 1951 & 1924 & 2322 & 2023 & 1883 & 1122.00 \\
\hline N4 & 1757 & 1441 & 920 & 1119 & 978 & 1115 & 1222 & 1443 & 1516 & 1512 & 1881 & 1873 & 2257 & 2009 & 1853 & 1093.00 \\
\hline N5 & 1828 & 1390 & 945 & 1117 & 1050 & 1068 & 1202 & 1344 & 1466 & 1393 & 1779 & 1778 & 1879 & 1880 & 1828 & 1063.00 \\
\hline N6 & 1859 & 1397 & 945 & 1101 & 1007 & 953 & 1188 & 1310 & 1435 & 1339 & 1748 & 1640 & 2005 & 1803 & 1814 & 1022.00 \\
\hline N7 & 1847 & 1429 & 921 & 1098 & 969 & 909 & 1156 & 1263 & 1532 & 1302 & 1949 & 1772 & 2148 & 1888 & 1815 & 992.00 \\
\hline N8 & 1805 & 1479 & 909 & 1085 & 961 & 859 & 1092 & 1197 & 1347 & 1396 & 1955 & 1812 & 1984 & 1919 & 1825 & 953.00 \\
\hline N9 & 1749 & 1425 & 907 & 1078 & 950 & 848 & 1096 & 1145 & 1596 & 1571 & 2000 & 1888 & 2008 & 1984 & 1820 & 917.00 \\
\hline N10 & 1662 & 1408 & 907 & 1046 & 947 & 844 & 1072 & 1059 & 1622 & 1503 & 1977 & 1916 & 2039 & 1989 & 1750 & 874.00 \\
\hline N11 & 1691 & 1415 & 866 & 1059 & 965 & 888 & 1057 & 1021 & 1437 & 1646 & 1951 & 1883 & 2060 & 2012 & 1726 & 864.00 \\
\hline N12 & 1675 & 1387 & 833 & 1122 & 970 & 938 & 1019 & 966 & 1459 & 1541 & 2108 & 1858 & 2044 & 2022 & 1692 & 822.00 \\
\hline N13 & 1657 & 1385 & 817 & 1138 & 974 & 843 & 1003 & 952 & 1621 & 1551 & 2113 & 1861 & 2062 & 1990 & 1666 & 793.00 \\
\hline N14 & 1629 & 1373 & 814 & 1060 & 1024 & 850 & 940 & 972 & 1651 & 1567 & 1994 & 1893 & 2035 & 1949 & 1629 & 778.00 \\
\hline N15 & 1644 & 1314 & 819 & 1126 & 1015 & 870 & 941 & 1115 & 1684 & 1691 & 2001 & 1934 & 2084 & 1911 & 1605 & 807.00 \\
\hline N16 & 1642 & 1305 & 825 & 1129 & 1073 & 966 & 1062 & 1143 & 1713 & 1696 & 2016 & 1978 & 2060 & 1908 & 1575 & 792.00 \\
\hline N17 & 1617 & 1331 & 822 & 1204 & 1061 & 998 & 1108 & 1132 & 1795 & 1670 & 1955 & 1959 & 2021 & 1728 & 1547 & 750.00 \\
\hline N18 & 1645 & 1378 & 841 & 1159 & 1090 & 1030 & 1127 & 1157 & 1837 & 1637 & 1922 & 1783 & 1853 & 1707 & 1494 & 710.00 \\
\hline N19 & 1645 & 1338 & 880 & 1203 & 1092 & 1020 & 1158 & 1205 & 1808 & 1441 & 1993 & 1756 & 1816 & 1719 & 1490 & 755.00 \\
\hline N20 & 1632 & 1421 & 960 & 1168 & 1072 & 948 & 1082 & 1245 & 1547 & 1472 & 1988 & 1756 & 1876 & 1737 & 1496 & 804.00 \\
\hline N21 & 1611 & 1456 & 1008 & 1191 & 1037 & 948 & 1093 & 1313 & 1712 & 1487 & 2011 & 1765 & 1918 & 1781 & 1503 & 832.00 \\
\hline N22 & 1700 & 1472 & 1024 & 1192 & 1037 & 1058 & 1264 & 1393 & 1761 & 1504 & 1995 & 1757 & 1915 & 1738 & 1507 & 863.00 \\
\hline N23 & 1700 & 1409 & 1004 & 1213 & 1059 & 1046 & 1245 & 1428 & 1721 & 1558 & 1920 & 1769 & 1923 & 1743 & 1535 & 923.00 \\
\hline N24 & 1678 & 1423 & 1022 & 1207 & 1007 & 1089 & 1270 & 1453 & 1790 & 1584 & 1932 & 1792 & 1917 & 1788 & 1571 & 1030.00 \\
\hline N25 & 1669 & 1456 & 1012 & 1191 & 1046 & 1071 & 1281 & 1493 & 1700 & 1587 & 1920 & 1733 & 1934 & 1788 & 1587 & 1032.00 \\
\hline N26 & 1661 & 1421 & 1009 & 1198 & 978 & 1074 & 1271 & 1477 & 1670 & 1586 & 1929 & 1814 & 1933 & 1798 & 1631 & 1062.00 \\
\hline $\mathrm{N} 27$ & 1677 & 1428 & 1000 & 1175 & 973 & 1058 & 1257 & 1480 & 1697 & 1610 & 1961 & 1828 & 1955 & 1814 & 1682 & 1065.00 \\
\hline N28 & 1645 & 1421 & 983 & 1174 & 1001 & 1085 & 1254 & 1492 & 1643 & 1590 & 1966 & 1863 & 2014 & 1854 & 1712 & 1082.00 \\
\hline N29 & 1642 & 1418 & 981 & 1157 & 947 & 1053 & 1255 & 1500 & 1663 & 1568 & 1942 & 1829 & 2019 & 1879 & 1744 & 1076.00 \\
\hline N30 & 1666 & 1440 & 974 & 1158 & 939 & 1079 & 1273 & 1498 & 1680 & 1570 & 1940 & 1777 & 1986 & 1900 & 1786 & 1070.00 \\
\hline N31 & 1629 & 1451 & 987 & 1162 & 934 & 1089 & 1293 & 1632 & 1672 & 1582 & 1916 & 1765 & 1971 & 1887 & 1830 & 1075.00 \\
\hline N32 & 1613 & 1534 & 1009 & 1152 & 939 & 1084 & 1307 & 1609 & 1646 & 1623 & 1911 & 1869 & 2032 & 1918 & 1847 & 1077.00 \\
\hline N33 & 1715 & 1540 & 1029 & 1154 & 962 & 1109 & 1313 & 1596 & 1826 & 1795 & 2043 & 1911 & 2059 & 2036 & 1864 & 1087.00 \\
\hline Var. & 9707 & 3088 & 5129 & 2121 & 2486 & 9088 & 13227 & 41985 & 15755 & 15279 & 5071 & 5854 & 14033 & 12125 & 19847 & 19723.00 \\
\hline STDV & 98 & 55 & 71 & 46 & 49 & 95 & 115 & 204 & 125 & 123 & 71 & 76 & 118 & 110 & 140 & 140.44 \\
\hline
\end{tabular}

\section{Results}

The proposed method was implemented on an LV dataset of 12 patients. The ED and ES of these data were determined by cardiologists from the National Heart Institute (IJN, KL. Malaysia), during a routineclinicalassessment. Using the current available software A-QLAB, the proposed method was implemented on the LV dataset, although the A-QLAB software was designed for quantifying the LV and mitral valve. The ED and ES Frames were determined manually by the expert during the routine clinical test for 12 patients. By default, the expert assumed the ED was the first frame of the cardiac cycle for all patients' data. The ES was determined by visual tracing of the cavity area changes and mitral valve together by considering the frame with the minimum area of the cavity and a closed mitral valve. The ED and ES frames that were selected by the experts and determined automatically by the proposed method were recorded and shown in Table 5. 
Table 4. Computed area for three slices and the average, the rows indicate the changes in the area of the segmented region of the frame $\rho$ compared with frame $\rho-1$

\begin{tabular}{llllll}
\hline $\begin{array}{l}\text { Normalized frame } \\
\text { (Indicator) } \rho\end{array}$ & $\begin{array}{l}\text { The frame position } \\
\text { in the cardiac cycle }\end{array}$ & $\begin{array}{l}\text { Segmented region } \\
\text { area of slice } 8\end{array}$ & $\begin{array}{l}\text { Segmented region } \\
\text { area of slice } 9\end{array}$ & $\begin{array}{l}\text { Segmented region } \\
\text { area of slice 10 }\end{array}$ & \begin{tabular}{l} 
Mean of area \\
\hline 1
\end{tabular} \\
\hline 1 & 1476 & 1160 & 1226 & $1248 \uparrow$ & 1732 \\
2 & 5 & $1401 \downarrow$ & $1173 \uparrow$ & 1225 & 1604 \\
3 & 9 & 1378 & 1111 & 1163 & 1508 \\
4 & 13 & 1396 & 1053 & 989 & 1480 \\
5 & 17 & 1258 & 933 & 865 & 1363 \\
6 & 21 & 1125 & 846 & 851 & 1262 \\
7 & 25 & 1062 & 771 & 834 & 1246 \\
8 & 29 & 1020 & 754 & 783 & 1229 \\
9 & 33 & 886 & 685 & 769 & 1188 \\
10 & 37 & $889 \uparrow$ & $693 \uparrow$ & $743 \downarrow$ & 1098 \\
11 & 41 & $826 \downarrow$ & $633 \downarrow$ & $702 \downarrow$ & 1262 \\
12 & 43 & $854 \uparrow$ & $649 \uparrow$ & $722 \uparrow$ & 1265 \\
13 & 47 & $836 \downarrow$ & $677 \uparrow$ & $825 \uparrow$ & 1429 \\
14 & 51 & $1036 \uparrow$ & $785 \uparrow$ & 896 & 1549 \\
15 & 55 & 1210 & 1171 & 990 & 1607 \\
16 & 59 & 982 & 971 & 1051 & 1582 \\
17 & 63 & 1314 & 1005 & & \\
\hline
\end{tabular}

Table 5. Determining the position of the ED and ES frame in the video of a cardiac cycle for 12 patients

\begin{tabular}{|c|c|c|c|c|}
\hline \multirow[b]{2}{*}{ Patient no. } & \multicolumn{2}{|c|}{ Manual (by expert) } & \multicolumn{2}{|c|}{ Automatic (proposed method) } \\
\hline & ED & ES & ED & ES \\
\hline$\overline{1}$ & 1 & 24 & 1 & 24 \\
\hline 2 & 1 & 34 & 2 & 34 \\
\hline 3 & 1 & 41 & 1 & 41 \\
\hline 4 & 1 & 31 & 5 & 31 \\
\hline 5 & 1 & 39 & 1 & 39 \\
\hline 6 & 1 & 25 & 1 & 25 \\
\hline 7 & 1 & 43 & 5 & 43 \\
\hline 8 & 1 & 37 & 1 & 37 \\
\hline 9 & 1 & 32 & 1 & 32 \\
\hline 10 & 1 & 58 & 1 & 58 \\
\hline 11 & 1 & 32 & 1 & 32 \\
\hline 12 & 1 & 35 & 1 & 35 \\
\hline
\end{tabular}

Table 6. Computing the EDA, to assess the effect of the variance in the frame number between the manual and the proposed automatic method. $e$ is the absolute difference between the EDA of experts and automatic method

\begin{tabular}{|c|c|c|c|c|c|c|c|}
\hline \multirow{3}{*}{$\begin{array}{l}\text { Frame detection } \\
\text { Method } \\
\text { Patient no. }\end{array}$} & \multicolumn{3}{|c|}{ Manual method } & \multicolumn{4}{|c|}{ Automatic method } \\
\hline & \multicolumn{3}{|l|}{ Cardiac stage } & \multicolumn{3}{|l|}{ Cardiac stage } & \multirow{2}{*}{$\begin{array}{l}\text { Absolute difference } \\
\text { between the EDA } \\
\text { of two methods (e) }\end{array}$} \\
\hline & ED frame no. & EDA & ES frame no. & ED frame no. & EDA & ES frame no. & \\
\hline$\overline{\mathrm{P} 1}$ & 1 & 94 & 24 & 1 & 99 & 24 & 5 \\
\hline $\mathrm{P} 2$ & 1 & 141 & 34 & 2 & 134 & 34 & 7 \\
\hline P3 & 1 & 130 & 41 & 1 & 121 & 41 & 9 \\
\hline P4 & 1 & 104 & 31 & 5 & 110 & 31 & 6 \\
\hline P5 & 1 & 98 & 39 & 1 & 100 & 39 & 2 \\
\hline P6 & 1 & 93 & 25 & 1 & 97 & 25 & 4 \\
\hline P7 & 1 & 81 & 43 & 5 & 87 & 43 & 6 \\
\hline P8 & 1 & 137 & 37 & 1 & 132 & 37 & 5 \\
\hline P9 & 1 & 89 & 32 & 1 & 93 & 32 & 4 \\
\hline P10 & 1 & 99 & 58 & 1 & 98 & 92 & 1 \\
\hline P11 & 1 & 86 & 32 & 1 & 86 & 32 & 0 \\
\hline $\mathrm{P} 12$ & 1 & 90 & 35 & 1 & 90 & 35 & 0 \\
\hline
\end{tabular}


From Table 5, it can be pointed out that the ES frames which were determined by the proposed method matches the ES frames which were selected by the experts for all of the 12 patients in the dataset.

\section{Discussion}

The result for ED frame selection is different between the proposed method and the experts by $2.5 \%$ of the data set (as in 2nd, 4th and 7th patients).

To analyse the effect of these difference in selecting the ED frames for these three patients P2, P5 and P7 using the proposed automatic method and the ED frame that selected manually by the experts for these three patients P2, P5 and P7. The absolute difference of the area of the cavity region in the End Dystonic frame (EDA) for both (automatic and manual) methods $(e)$ is computed as declared in Table 6 . Then the cavity volume at the ED is computed for the cases that have an error in detecting the ED frame for the patients $\mathrm{P} 2$, $\mathrm{P} 5$ and $\mathrm{P} 7$ is $0.05 \mathrm{~mL}, 0.077 \mathrm{~mL}$ and $0.04 \mathrm{~mL}$ respectively, regarding to the $\mathrm{LV}$ volume at EndDiastolic frame (EDV $=120 \mathrm{~mL}$ ) according to (Schlosser et al., 2005) and (Blalock et al., 2013) this values represent $0.4,0.6$ and $0.3 \%$ of the EDV volume, which is a very small value and can be neglected.

\section{Conclusion}

This research is presented to explain the proposed algorithm of the automatic ED and ES frame detection from one cardiac cycle based on the medical definition of the ED as the maximum volume of the cavity and the ES as the minimum cavity volume in the cardiac cycle. By computing the segmented area for three slices in the middle region of the RV cavity, this region is selected after analysing the changes in the cavity area in three regions of the cavity Inflow, Middle and Outflow regions. The middle region was the most significant part in the changing area along a cardiac cycle and not the inflow and outflow tract. That is because the area of the free wall in this region is wider than the area of the free wall in the inflow and outflow tract, as illustrated in Fig. 2.

The cavity region is segmented for the three slices, along the video of one cardiac cycle and then the average of the slice area is computed. The frames of the maximum area and minimum area are detected as the ED and ES frames respectively. The results of the proposed algorithm have been validated using the AQLAB system and datasets of different patients.

\section{Funding Information}

The authors have no support or funding to report.

\section{Author's Contributions}

All authors equally contributed in this work.

\section{Ethics}

This article is original and contains unpublished material. The corresponding author confirms that all of the other authors have read and approved the manuscript and no ethical issues involved.

\section{References}

Aune, E., M. Baekkevar, O. Rodevand and J.E. Otterstad, 2009. The limited usefulness of realtime 3-dimensional echocardiography in obtaining normal reference ranges for right ventricular volumes. Cardiovascular Ultrasound, 7: 35-35. DOI: 10.1186/1476-7120-7-35

Barash, P.G., B.F. Cullen, R.K. Stoelting, M.C. Stock and M.K. Cahalan, 2011. Clinical anesthesia. 1st Edn., Lippincott Williams and Wilkins, ISBN-10: 1451122977, pp: 1760.

Blalock, S., F. Chan, D. Rosenthal, M. Ogawa and D. Maxey et al., 2013. Magnetic resonance imaging of the right ventricle in pediatric pulmonary arterial hypertension. Pulmonary Circulat., 3: 350-355. DOI: $10.4103 / 2045-8932.114763$

Chua, S., R.A. Levine, C. Yosefy, D.M. Handschumacher and J. Chu et al., 2009. Assessment of right ventricular function by realtime three-dimensional echocardiography improves accuracy and decreases interobserver variability compared with conventional two-dimensional views. Eur. J. Echocardiography, 10: 619-624. DOI: 10.1093/ejechocard/jep013

Crean, A.M., N. Maredia, G. Ballard, R. Menezes and G. Wharton et al., 2011. 3D Echo systematically underestimates right ventricular volumes compared to cardiovascular magnetic resonance in adult congenital heart disease patients with moderate or severe RV dilatation. J. Cardiovascular Magnetic Resonance, 13: 78-78. DOI: $10.1186 / 1532-429 X-13-78$

Dawood, T., M.P. Schlaich, N. Straznicky, M. Grima and C. Ika-Sari et al., 2011. Renal denervation: A potential new treatment modality for polycystic ovary syndrome. J. Hypertens., 29: 991-996. DOI: 10.1097/HJH.0b013e328344db3a

Darvishi, S., H. Behnam, M. Pouladian and N. Samiei, 2012. Measuring left ventricular volumes in twodimensional echocardiography image sequence using level-set method for automatic detection of enddiastole and end-systole frames. Res. Cardiovasc Med., 1: 39-45. DOI: 10.5812/cardiovascmed.6397 
Gifani, P., H. Behnam, A. Shalbaf and Z.A. Sani, 2010. Automatic detection of end-diastole and end-systole from echocardiography images using manifold learning. Phy. Measure., 31: 1091-1091. DOI: $10.1088 / 0967-3334 / 31 / 9 / 002$

Gopal, A.S., O. Ebere, C.J. Chizor, I.S. Alan, K. S. Rena and T.W. Schapiro et al., 2007. Normal values of right ventricular size and function by real-time 3-dimensional echocardiography: Comparison with cardiac magnetic resonance imaging. J. Am. Soci. Echocardiography, 20: 445455. DOI: 10.1016/j.echo.2006.10.027

Hussein, S.M., N.N. Batada, S. Vuoristo, R.W. Ching and R. Autio et al., 2011. Copy number variation and selection during reprogramming to pluripotency. Nature, 471: 58-62. DOI: 10.1038/nature09871

Klabunde, R. 2011. Cardiovascular physiology concepts. Cardiovascular physiology concepts. 1st Edn., Illustrated, Lippincott Williams and Wilkins, Philadelphia, ISBN-10: 1451113846, pp: 243.

Niemann, P.S., L. Pinho, T. Balbach, C. Galuschky, M. Blankenhagen and M. Silberbach et al., 2007. Anatomically oriented right ventricular volume measurements with dynamic three-dimensional echocardiography validated by 3-tesla magnetic resonance imaging. J. Am. Coll. Cardiol., 50: 16681676. DOI: 10.1016/j.jacc.2007.07.031

Nosir, Y.F.M., P.M. Fioretti, W.B. Vletter, B.E. Boersma and A. Salustri et al., 1996. Accurate measurement of left ventricular ejection fraction by three-dimensional echocardiography a comparison with radionuclide angiography. Circulation, 94: 460-466. DOI: 10.1161/01.CIR.94.3.460

Ostenfeld, E., M. Carlsson, K. Shahgaldi, A. Roijer and J. Holm, 2012. Manual correction of semi-automatic three-dimensional echocardiography is needed for right ventricular assessment in adults; validation with cardiac magnetic resonance. Cardiovascular Ultrasound, 10: 1-1. DOI: 10.1186/1476-7120-10-1
Schlosser, T., K. Pagonidis, C.U. Herborn, P. Hunold and K.U. Waltering et al., 2005. Assessment of left ventricular parameters using 16-MDCT and new software for endocardial and epicardial border delineation. Am. J. Roentgenol., 184: 765-773. DOI: 10.2214/ajr.184.3.01840765

Shalbaf, A., H. Behnam, P. Gifani and Z. AlizadehSani, 2011. Automatic detection of end systole and end diastole within a sequence of 2-D echocardiographic images using modified Isomap algorithm. Proceedings of the 1st Middle East Conference on Biomedical Engineering, Feb. 2124, IEEE Xplore Press, Sharjah, pp: 217-220. DOI: 10.1109/MECBME.2011.5752104

Umberto, B., M. Davide and S. Ovidio, 2008. Automatic computation of left ventricle ejection fraction from dynamic ultrasound images. Patt. Recognit. Image Anal., 18: 351-358. DOI: $10.1134 / \mathrm{S} 1054661808020247$

Yuanfang, G., V. Yao, K. Tsui, M. Gebbia and M.J. Dunham et al., 2011. Nucleosome-coupled expression differences in closely-related species. BMC Genom., 12: 466-466. DOI: 10.1186/1471-2164-12-466

Zhu, Y., X. Papademetris, J.S. Albert and S.D. James, 2009. A Dynamical Shape Prior for LV Segmentation from RT3D Echocardiography. In: Medical Image Computing and Computer-Assisted Intervention-MICCAI, Yang, G.Z., D. Hawkes, D. Rueckert, A. Noble, C. Taylor (Eds.)., Springer Berlin Heidelberg, ISBN-10: 978-3-642-04267-6, pp: 206-213. 